XVIIl.

\title{
477 „zentralnormale“ Ist- und Formelgewichte erwachsener Menschen.
}

\author{
Von
}

Dr. med. Gustav Oeder, Diätkuranstalt Niederlössnitz bei Dresden.

In der Berliner klin. Wochenschr., 1915, Nr. 17/18, habe ich meine ersten Mitteilungen aus den Jahren 1909-1911 über das nzentralnormale Körpergewicht Erwachsener (= mittelste Stufe zwischen Magerkeit und Fettleibigkeit) und seine praktisch-einfache Berechnungsmöglichkeit aus der nproportionellen "Körpergrösse durch Veröffentlichung weiterer 281 "zentralnormaler" Fälle zu unterstützen gesucht und habe dabei auch die Genauigkeit und Häufigkeit in der Uebereinstimmung meiner Formelgewichte mit den zugehörigen zentralnormalen Istgewichten zahlenmässig im einzelnen, an Durchschnitten und an Kurven gezeigt. Die von mir wiederholt hervorgehobene praktische Brauchbarkeit meiner empirischen Berechnungsformeln für das "zentralnormale" Körpergewicht hat auf Grund eigener Nachprüfung meines Wissens bisher nur Zickgraf (Med. Klin., 1912, Nr. 32) ausdrücklich bestätigt; offen bestritten hat sie Niemand. Doch habe ich die Empfindung, dass gegen diese Formeln noch ein "latentes" Misstrauen besteht, welches vielleicht nicht nur auf einem verschiedensinnigen Gebrauch des Wortbegriffes „zentralnormal", sondern auf einer Art ästhetischen Unbehagens mit wissenschaftlicher Färbung beruht gegenüber allem, was nach "roher" Empirie aussieht. Es will mir sogar fast scheinen, als ob man an manchen Stellen mehr, als die unverhüllte Darstellung der nicht immer schönen "rohen" Wirklichkeit, ihre Einwicklung in ein von aussen hübsch "exakt" ansprechendes, theoretisches Mäntelchen geliebt habe, dem man gar zu gern den Namen "Voraussetzungslosigkeit" gab, während man dieses Mäntelchen stillschweigend mit ungeprüften Voraussetzungen im Innern um so reichlicher unterfütterte. Nolens volens muss man daher immer und immer wieder die unverhüllte Wirklichkeit herausstellen, soweit sie zahlenmässig erfassbar ist, gegebenenfalls auch stillschweigend gemachte Voraussetzungen, und zwar schon bei den Untersuchungsmethoden, an ihr zahlenmässig nachprüfen. Nun habe ich gerade eine ungefähr gleich grosse Personenzahl wie 1915, nämlich 288 "zentralnormal" genährte verschiedene Personen, und zwar 133 männliche und 155 weibliche, mit insgesamt 477 lstgewichten beisammen. Deshalb scheint es mir an der Zeit und geboten, wieder eine Mitteilung darüber zu machen. Allerdings unterscheidet sich mein hier zusammengestelltes Studienmaterial von dem früberen dadurch, dass die inspektorische Feststellung der Besichtigungsmerkmale der "zentralnormalen" Ernährungszustandsstufe 


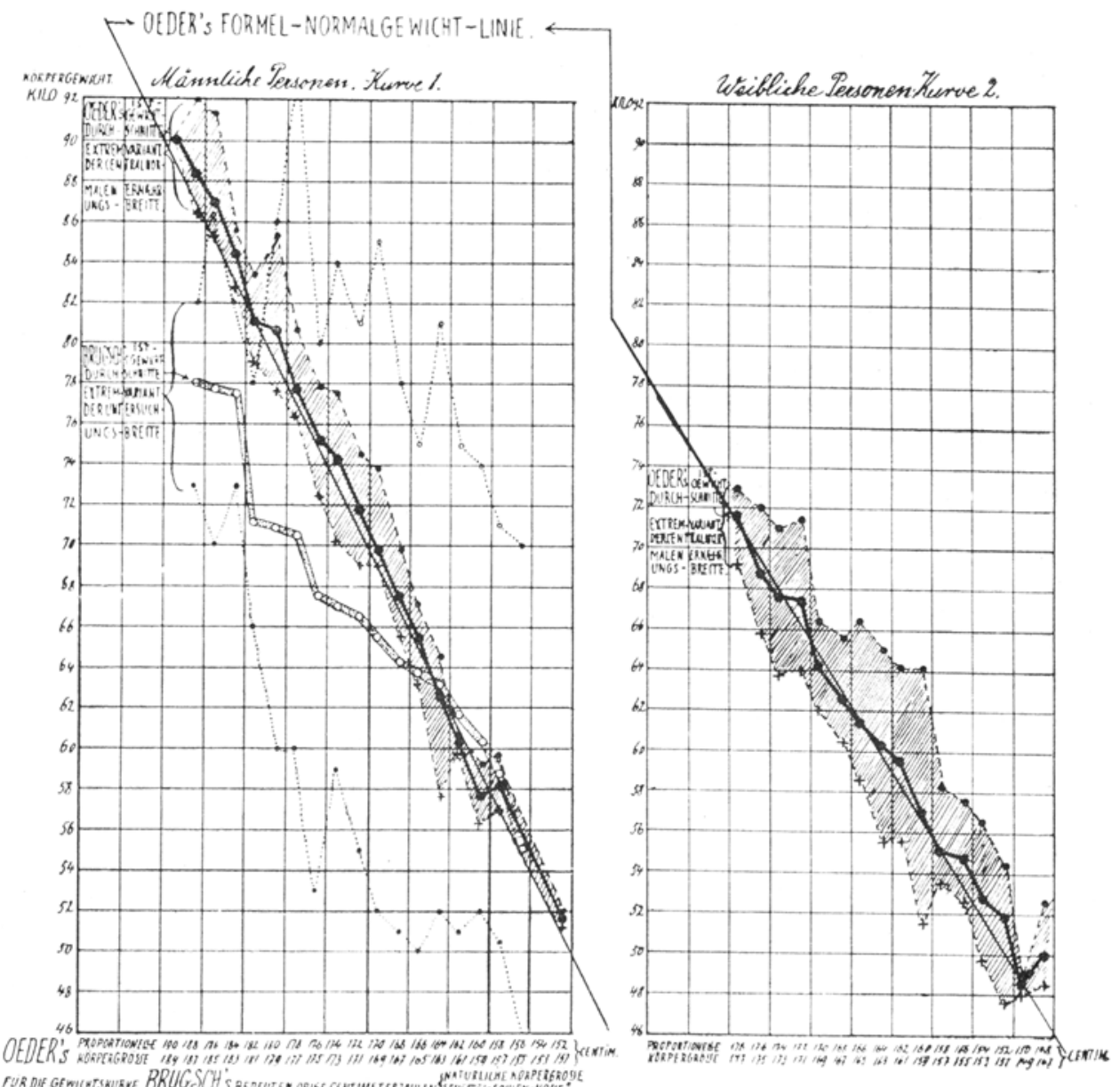

Anmerkung: Oeder's "Istgewichtsdurchschnitte" sind aus sämtlichen physiologischen "zentraluormalen" Einzelgewichtsvarianten der einzelnen „proportionellen " Körpergrössengruppen nach den in den weggefallenen Tabellen verzeichneten Zahlen berechnet. Die "zentralnormale" Ernährungsbreite (gekennzeichnet durch Schraffierung der zwischen den Extrenvarianten gelegenen Fläche) ist bei den männlichen Körpergewichten etwas schmaler, als bei den weiblichen; doch darf dabei darauf hingewiesen werden, dass die extremen Lussenscitcr dor weiblichen Körpergewichte fast immer nur vereinzelt waren. Oeder's "Formelnormalgewichtslinie“ schliesst sich ersichtlich sehr eng dem Verlauf der Linie an, welche die .zentralnormalen Istgewichtsdurchschnitte ${ }^{*}$ verbindet. Zum Vergleich sind die Brugsch'schen Linien eingezeichnot. Die Linie der Brugsch'schen Istgewichtsdurchschnitte verläuft von denen der Körpergrössengruppe $164 / 163 \mathrm{~cm}$ an weit unterhalb der Istgewichtslinio Oeder's. Allerdings ist ein richtiger Vergleich beider Linien unmöglich, weil die Körperlängen Brugsch's und Oeder's ungleichartige sind. Die Brugsch'sche Untersuchungsbreite ist aber unverkennbar sehr viel breiter, wie die Oeder'sehe ,zentralnormale" Ernährungsbreite bei den männlichen Personen (weibliche standen aus dem Brugsch'schen Material zum Vergleich nicht zur Verfügung). Die Brugsch'sche Breite muss also vermutlich stark ungleich genährtes Material enthalten haben. Die Brugsch'schen Istgewichtsdurchschaitte dürfen von der Körpergrössengruppe $164 / 163 \mathrm{~cm}$ an wohl mit grosser Wahrscheinlichkeit als Körpergewichte angesproehen werden, die einem mehr oder minder "mageren" Ernährungszustand entsprechen, soweit dabei völlig erwachsene Personen in Betracht kommen. 
dieses Mal nicht bei allen Personen ganz so genau hat erfolgen können, wie bei dem früheren, welches besonders sorgfältig auf das Vorhandensein des zentralsten Grades der zentralnormalen Stufenbreite beobachtet worden war. Diesmal sind mehr gelegentliche Feststellungen getroffen worden, wobei es nicht regelmässig geglückt ist, gerade den zentralsten Grad stets zu erwischen und das zugehörige Istgewicht $\mathrm{zu}$ wägen, weil eben nur gelegentlich und in grösseren, auch unregelmässigeren, zeitlichen Zwischenräumen die unumgängliche Nacktbesichtigung hat vorgenommen werden können, um Belästigung der Kranken nach Möglichkeit zu vermeiden; auch sind dicsmal einige Istgewichte zeitweise pathologisch beeinflusster Personen mit darunter, die ich werde ausscheiden müssen. Immerhin ist es trotz allem gelungen, noch ein so gleichmässiges Studienmaterial zu erhalten, dass auch an den dabei festgestellten Istgewichten meine Formelgewichte auf ihre "Stimmigkeit" geprüft werden können; ja in einer Hinsicht ist das folgende Material sogar noch lehrreicher als das frühere: Während ich nämlich früher für jede Person nur ein einziges lstgewicht, und zwar das Höchstgewicht desjenigen Tages genommen hatte, an welchem die Naktbesichtigung das Vorhandensein der zentralnormalen Besichtigungsmerkmale am vollkommensten ergeben hatte, habe ich diesmal alle, auch die nur als „annähernd zentralnormal" beurteilten Körpergewichte aufgeführt; so habe ich bei einzelnen Personen 2-9, oft verschieden grosse, zentralnormale Istgewichte notiert. Die Einreihung der Einzelfälle in die folgende Tabelle ${ }^{1}$ ) ist nach der "proportionellen" Körpergrösse in Einzentimeterstufen erfolgt; doch habe ich jeweils die "natürlichen" Scheitelsohlenhöhen daneben beschrieben. Die „proportionellen " Körpergrössen geben bei meinen Männern von $152-190 \mathrm{~cm}$, bei den Frauen von 147 bis $178 \mathrm{~cm}$, die "natürlichen" Scheitelsohlenhöhen bei den Männern von $150-191 \mathrm{~cm}$, bei den Frauen von $144-174 \mathrm{~cm}$. Das Jebensalter schwankte vom 24.-70. Lebensjahr. Die Formelgewichte sind nach meinen männlichen und weiblichen Formeln (vgl. Berliner klin. Wochenschrift, 1915, Nr. 17/18) berechnct. Die Istgewichte sind wieder Tageshöchstgewichte (ohne Kleidung). Ausrufungszeichen (!) neben den Istgewichten zeigen pathologische Einflüsse an. Die zur gleichen Person gehörigen Zahlen sind durch Abstrich unter den Körpergrössenzahlen gekennzeichnet. Alles Uebrige sagen die Spaltenüberschriften (vgl. Kurven statt Tabellen!).

Aus den Tabellen mache ich hier folgende Zusammenstellungen der gemischt pathologisch-physiologischen und der rein physiologischen Körpergewichte:

1) Anmerkung: Diese Arbeit wurde bereits am 22.4. 1918 zur Veröffentlichung angenommen; nachträglich verlangt der Verlag jetzt der hohen Druckkosten wegen die Ersetzung der Tabellen mit den ursprünglichen Körpergewichts- und KörperlängenEinzelzahlen durch Kurventafeln, welche zur Vermeidung unübersichtlicher Detailierung und Raumbeanspruchung nur unter Zusammenfassung der Einzentimeterlängen zu Gruppen und nur mit Gewichtsdurchschnitt- und Extremvarianten-Zahlen herstellbar sind, wodurch die ursprüngliche Nachprüfungsmöglichkeit der Einzelzahlen leider zum Teil verloren geht.

Der Verfasser. 
I. Die Summen aller Istgewichte und Formelgewichte:

\begin{tabular}{|c|c|c|c|c|}
\hline & \multicolumn{2}{|c|}{ Istgewichte } & \multicolumn{2}{|c|}{ Formelgewicbte } \\
\hline & $\begin{array}{l}\text { (mit Einschluss der } \\
32 \text { pathol.) }\end{array}$ & $\begin{array}{l}\text { mit Ausschluss der } \\
32 \text { pathol. }\end{array}$ & $\begin{array}{c}\text { nach "proportionellen" } \\
\text { Kö̈rpergrössen }\end{array}$ & $\begin{array}{l}\text { [nach Scheitel- } \\
\text { soblenböhen] }\end{array}$ \\
\hline \multirow[t]{2}{*}{$\begin{array}{l}\text { Männl. Personen } \\
\text { Weibl. Personen }\end{array}$} & $\begin{array}{l}(18336,5 \mathrm{~kg} 251 \text { (Gew.) } \\
(13522,7 \mathrm{~kg} 226 \text { Gew. }\end{array}$ & $\begin{array}{l}16491,6 \mathrm{~kg} \\
13026,5 \mathrm{~kg} \\
(218 \mathrm{Gew} .)\end{array}$ & $\begin{array}{l}(18310,0 \mathrm{~kg}) 16497,0 \mathrm{~kg} \\
(1347 \tau, 6 \mathrm{~kg}) 12992,1 \mathrm{~kg}\end{array}$ & {$\left[\begin{array}{l}{[16086,5 \mathrm{~kg}]} \\
{[12595,2 \mathrm{~kg}]}\end{array}\right.$} \\
\hline & $(31859,2 \mathrm{~kg} 477$ (iew) & $29518,1 \mathrm{~kg}$ (445 Gew.) & $(31787,6 \mathrm{~kg}) \mathbf{2 9 4 8 9}, 1 \mathrm{~kg}$ & {$[28681,7 \mathrm{~kg}]$} \\
\hline
\end{tabular}

\begin{tabular}{l|c|c}
\hline & \multicolumn{2}{|c|}{ Unterschiede der nich tpathologischen Ist- und Formelgewichte } \\
\cline { 2 - 3 } & aus ,proportioneller" Körpergrösse & [aus Scheitelsoblenhöhe] \\
\hline Männl. Personen. . . & $+5,4 \mathrm{~kg}$ & {$[-405,1 \mathrm{~kg}]$} \\
Weibl. Personen. . & $-34,4 \mathrm{~kg}$ & {$[-431,3 \mathrm{~kg}]$} \\
\hline & $-\mathbf{2 9 , 0} \mathrm{kg}$ & {$[-836,4 \mathrm{~kg}]$}
\end{tabular}

Ich will nur die physiologischen Istgewichtssummen (fett gedruckt!) mit den zugehörigen Formelgewichtssummen vergleichen. Diese Summen der Istgewichte stimmen mit den Formelgewichten überein: bei deren Berechnung aus "proportionellen" Körpergrössen a) für die männlichen Personen auf $+5,4 \mathrm{~kg}(!)$, um welche die Formelgewichte grösser sind; b) für die weiblichen Personen auf $-34,4 \mathrm{~kg}$, um welche die Formelgewichte kleiner sind. Die "Stimmigkeit" ist bei den männlichen Formelgewichtssummen etwas besser, als bei den weiblichen; das gleiche Verhalten war auch bei meinen früheren Zusammenstellungen vorhanden; die männliche Formel muss also genauer sein, wie die weibliche, obgleich bei den männlichen der Brustumfang nicht zur Berechnung gezogen ist, während er bei der weiblichen in der Formelberechnung berücksichtigt ist. Doch sind die Untersehiede der Ist- und Formelgewichtssummen bei jedem Geschlecht nur klein, und zusammengerechnet betragen sie im Durchsehnitt nur 29,0:29518,1=0,00098 kg $=0,98$ oder rund nur $\mathbf{l}$ p $M !$ Ganz anders verhält es sich mit den Formelgewichten, die aus den "natürlichen" Scheitelsohlenhöhen gefunden sind (in [] Klammern), selbst wenn sie im übrigen nach den gleichen Formeln, wie die aus "proportionellen" Körpergrössen, berechnet werden. Einfach der Einsatz der "natürlichen" statt der "proportionellen" Körpergrösse in meine Formeln hat einen Gewichtsunterschied in den Summen zwischen Ist- und Formelgewichten bei den gleichen Personen von $836,4 \mathrm{~kg}(!)=28,3 \mathrm{pM}$. zur Folge. Das ist gegenüber dem Obigen ein Unterschied von über 28 facher Grösse! Daraus ergibt sich einwandsfrei die praktische Ueberlegenheit der Berechnung des zentralnormalen Körpergewichts nach meinen Formeln aus der "proportionellen" gegenüber der aus der "natürlichen" Körpergrösse.

II. Die Durchschnittszahlen ergeben naturgemäss das gleiche Bild, wie die Summenzahlen, nur in kleineren Zahlenwerten. Ich berïcksichtige auch hierbei nur die physiologischen Istgewichte. Diese Durchschnitte ergeben für die Istge wichte und F ormelge wich te Unterschiede: 
a) bei den männlichen Personen mit 227 Einzelgewichten $72,650 \mathrm{~kg}$ Istgewicht, $72,674 \mathrm{~kg}$ Formelgewicht $=+0,024 \mathrm{~kg}$ oder $24 \mathrm{~g}$; b) bei den weiblichen Personen mit 218 Einzelgewiehten $59,755 \mathrm{~kg}$ Istgewicht, $59,597 \mathrm{~kg}$ Formelgewicht $=-0,158 \mathrm{~kg}$ oder $158 \mathrm{~g}$. Währond hier der Durchschnittsunterschied zwischen Ist- und Formelgewichtsdurchschnitt für die männlichen Personen ganze $24 \mathrm{~g}$, für die weiblichen $158 \mathrm{~g}$ nur beträgt, würde er bei den aus der "natürlichen" Schcidelsohlenhöhe berechneten Formelgewichten (männl. 70,866, weibl. $57,776 \mathrm{~kg}$ ) $-1,784 \mathrm{~kg}$ bzw. $1,979 \mathrm{~kg}$ ausmachen. Also auch bei der Durchschnittsrechnung ist die Ueberlegenheit der „proportionellen" gegenüber der "natürlichen" Körpergrösse als Berechnungslänge zahlenmüssig unverkennbar. Noch deutlicher aber wie aus der Genauigkeit in der Uebereinstimmung der Summen und Durchschnitte erhellt die praktische Richtigkeit meiner mit Hilfe der "proportionellen" Körpergrösse berechneten Formelgewichte aus der Häufigkeit, in welcher die einzelnen Formelgewichte innerhalb einer bestimmten Genauigkeitsbreite mit den zentralnormalen lstgewichten übereinstimmen. Aus früher bereits dargelegten Gründen habe ich ursprünglich \pm 3 bis $4 \mathrm{~kg}$ als für den Einzelfall zulässigen Gewichtsunterschied bei meinen Vergleichsprüfungen angenommen; ich wähle für die jetzige Prüfung $\pm 3,5 \mathrm{~kg}$ als Genauigkeitsgrenze; die Begründung dafür werde ich am Schluss geben. Dabei stimmen

III. alle aus der „proportionellen " Körpergrösse formelmässig errechneten Einzelgewichte mit don gewonnenen Einzelgewichten auf $\pm 3,5 \mathrm{~kg}$ überein: a) für die mänulichen Personen unter 227 Gewichtszahlen 216 mal, d. h. in 95,16 pCt. aller physiologischer Fälle; b) für die weiblichen Personen unter 218 Gewichtsahlien $210 \mathrm{mal}$, d. h. in $96,33 \mathrm{pCt}$. aller physiologischer Fälle.

Ist auch die "Stimmigkeit" in den Summen und Durchschnitten der vorliegenden Zusammenstellung nicht ganz so genau, wie in denen meiner früheren, so ist doch die Häufigkeit der Uebcreinstimmung bei den Einzelgewichten annähernd die gleiche (früher aul $\pm 3,0 \mathrm{~kg}$ in 94,7 pCt. bzw. 91,28 pCt.; auf $\pm 4,0 \mathrm{~kg} 97,73$ pCt. bzw. 95,98 pCt.).

Wer den Grad der Uebereinstimmung in Gewichtssummen und - Durchschnitten, sowie den Grad und die Häufigkeit der Vebereinstimmung in den Einzelgewichten bei allen Körpergrössen zahlenmässig auf sich wirken lässt, wird sich der brutalen Gewalt dieser Zahlen gar nicht entziehen können. Diese Zahlen zwingen absolut, sowohl die tatsächliche Richtigkeit meiner empirischen Berechnungsformeln und damit die Gesetzmässigkeit der Beziehungen zwischen nzentralnormalem" Istgewicht und "proportioneller" Körpergrösse bis auf Beweis des Gegenteils anzuerkennen. Theoretische Bedenken irgendwelcher Art brauchen also von der Anwendung der gleichen Formeln und auch von ihrer Nachprüfung nicht abzuhalten. Wenn 'Th. Brugseh' vor kurzem die Beziehungen der "Körpergewichte" zur "Körperlänge" gelegentlich seiner dankenswerten anthropologischen Untersuchungen an männlichen Personen zwischen dem 22.-28. Luebensjahr nebenbei ge-

1) Theodor Brugsch, Masse und Proportionen zur Charakteristik des Indi. viduums in seinem Habitus. Zeitschr. f. experim. Path. u. Ther. 1917. Bd. 19. H. 1. 
prüft und zu dem Ergebnis kommen zu sollen vermeint hat: „auf eine für alle Isängen giltige Formel lassen sie sich nicht bringen", so kann er zu diesem Urteil nur auf Grund eines dafür ungeeigneten Studienmaterials und unter zeitweiser $\Lambda$ usserachtlassung des Prüfungsgrundsatzes "ceteris paribus" gelangt sein. Ungeeignet erscheint mir sein Material, weil "noch nicht völlig Erwachsene" mit "Erwachsenen" zusammengestellt sind, und weil die Ernährungszustandsstufe seiner Einzelfälle nicht die gleiche, geschweige denn eine „zentralnormale" war. Die B.'sche Zufalls-Gewicht-Varianten-Breite Gleichgrosser ist viel zu ausgedehnt und bei den einzelnen Körperlängenstufen viel zu ungloich. Das letztere berührt zweifellos auch den Grundsatz "ceteris paribus"; denn wer die grossen Gewichtsunterschiede kennt, welche allein durch die Verschiedenheit der Ernährungszustandsstufen bei gleichgrossen Personen und im gleichen Einzelfall erzeugt werden können, der wird sie "ceteris paribus" berücksichtigen müssen; er wird nicht im Ernährungszustand indifferenzierte Personen mit ihren Körpergewichten zusammenstellen dürfen; er wird vielmehr die Gesamtbreite des Ernährungszustandes in Stufen einteilen und die dazugehörigen Gewichtsabstufungen umgrenzen müssen. Wer weiter sich erinnert, dass die sogen. menschliche "Körperlänge" doch nicht die Länge eines in seinen natürlichen Einzelabschnitten beim Einzelfall und bei verschiedenen Personen gleichdicken und gleichbreiten gleichmässigen Körpers ist, der wird auch die Verschiedenheit der Einzelabschnitte bei der Jjängenmessung berïcksichtigen müssen; zum mindesten wird er die anthropologisch ancrkannte Zweiteilung der Gesamtlänge in eine "Ober-" und "Unterlänge" zu beachten haben. Gar wenn er beide Maasse (Gewicht und Länge) dieser Körper zu einander in Beziehung setzen und nach gesetzmässigen Beziehungen der Körpermasse zur Körperlänge fałnden will, wird er um die Prüfung der Voraussetzungen für die Anwendung der Wägung, Längen- und Umfangmessung als Messmethoden und um die Berücksichtigung der verschiedenen Einflüsse auf das Gewicht und die Länge nicht herum kommen. Versucht er es anders, so wird er sich nicht wundern dürfen, dass er "typische" Beziehungen nicht finden kann.

Bezüglich der Gewichte kann ich es zwar dahingestellt sein lassen, ob die B.'schen Körpergewichte sogen. „Kriegsnotgewichte" kriegsmässig unterernährter Personen waren. Wenn man aber aus der Zeit der B.'schen Veröffentlichung (Herbst 1917?) und aus folgender Erwägung eine Vermutung entnehmen wollte, so würde sie für durchschnittliche ${ }_{n}$ Kriegsmagerkeit" sprechen. Die öffentliche Nahrungsrationierung nach dem sogen. "Durchschnittskopt", welche von Anfang an die gleiche Ration für die verschieden grossen Erwachsenen bestimmt hat, musste für den Ernährungszustand der Betroffenen ceteris paribus die Wirkung haben, und hat sie bei Einhaltung der Rationierung tatsächlich auch gehabt, dass die kleinsten Menschen ebenso viel erhielten, wie die grössten. Da aber vom September 1916 an ausserdem die "Prokopfration" zur Erhaltung des Körpermassenbestandes bei Erwachsenen überhaupt nicht mehr zureichte, so mussten alle, die sich nebenher nichts beschaffen konnten (z. B. Lazarettinsassen), durch Einschmelzen ihrer Körpersubstanz. abmagern und an Körpergewicht abnehmen, die Grossen mehr 
und schneller, als die Kleinen. Die notwendige Folge hiervon musste sein, dass zur gleichen Untersuchungszeit die Grossen ein relativ stärker vermindertes Körpergewicht aufwiesen, als die Kleinen. In der Tat zeigt nun die B.'sche Gewichtstabelle von Soldaten aus der fraglichen Kriegszeit, dass die Durchsehnittsgewichte der "Grossen" im Verhältnis zu ihror Körpergrösse jeweils kleiner waren, als die der "Kleinen". B. sucht das formelmässig dadurchauszudrücken, dass er bei seinen kleinsten Personen, dic wenig oder gar nichts abgenommen zu haben brauchten, die Brocaschen Formelgewichte als zutreffend crklärte ( $=$ Körpergrösse in $\mathrm{cm}$ - 100), bei seinen grössten (Abgemagertsten) aber das Broca-Formelgewicht $-10 \mathrm{~kg}$ einsetzte. Seine ${ }_{\pi}$ Grössten “ waren also im Durchschnitt um $10 \mathrm{~kg} \mathrm{zu}$ mager. Allein - wie gesagt - es ist an sich ganz gleichgültig, ob die Kriegsrationierung an dem Mindergewicht der Grossen schuld war oder nicht: "mager" war im Durchschnitt der Ernährungszustand der B.'schen Grossen allemal, wenn auch vielleicht nicht "kriegsmager". Ungleich genährt waren die Personen aller einzelnen B.'schen Körpergrössenstufen ebenfalls; denn die Gewichtsunterschiede betrugen in jeder Körpergrössenstufe 9--33 (!) kg, im Durchschnitt $22,8 \mathrm{~kg}$. So grosse durchschnittliche Körpergewichtsunterschiede gleichgrosser Gleichgeschlechtiger sind ohne Verschiedenheit des Ernährungszustandes schlechterdings unmöglich; Muskel- und Knochenunterschiede machen vielloicht ausnahmsweise einmal, aber nie im Durchschnitt gleichgrosser Gleichgeschlechtiger $10 \mathrm{~kg}$ aus; das haben mir zahlreiche Sonderusammenstellungen gezeigt, wobei ich nicht nur nach „proportionellen" Körpergrössen, sondern auch nach "Scheitelsohlenhöhen“ verglichon habe. Iah glaube auch nicht, dass B. Gegenteiliges unter Vorbringung von beweiskräftigem Material behaupten oder dio Beeinflussung des Körpergewichts durch Veränderung im Ernährungszustand in Abrede stellen möchte. Er hat den Einfluss der Ernährungszustandsverschiedenheiten auf die Körpergewichte seines Materials aber einfach ungeprüft gelassen und aus den ungleichartigen Körpergewichten der Einzelpersonen jeder Grössengruppe "Durchschnittsgewichto ${ }^{4}$ berechnet, die er meinte, als "normotypische“ bewerten zu dürfen. Er sagt ausdrücklich: „Diese Durchschnittsgewichte können für Normalgewichte angesehen werden." In Wirklichkeit sind solche Durchschnittsgewichte von ungleich genährten Einzelpersonen nur "Zufallsmischungsdurehschnitte". "Zufälliges" nennt man aber wohl kaum "normal"! Was alsdann die B.'schen Körperlängen" angeht, so sind es die sogen. "Scheitelsohlenhöhen", die ich als zum Studium der Beziehungen zwischen ${ }_{n}$ Körperlänge" und "Körpergewicht" ungeeignet bezeichnen muss, weil die Differenzierung der Gesamtlängen zum mindesten in ${ }_{n}$ Ober- "und „Unterlängen" und der Einfluss der Verschiedenheit der Oberlängen (selbst bei gleicher Seheitelsohlenhöhe) auf das dabei vorhandene verschieden grosse Körpergewicht ceteris paribus ungeprüft blieb. Ebenso ist der Einfluss der Verschiedenheit des Ernährungszustandes (bzw. Körpergewichts) auf den Brustumfang von B. nicht nachgeprüft worden. Und doch beträgt nach Sonderzusammenstellungen, welche ich gemacht habe, der Brustum fangsunterschied bei der gleichen Person, soweit er durch Ernährungsgewichts- 
unterschiede bedingt ist, auf $23 \mathrm{~kg}$ Gewichtsunterschied bei $171 \mathrm{~cm}$ Scheitelsohlenhöhe etwa 9-11 cm! Er ist also etwa ebenso gross, wie der ganze Brustumfangsunterschied zwischen den Eng- und Weitbrüstigen B.'s, der bei der gleichen Körperlängengruppe nur etwa $10 \mathrm{~cm}$ zu betragen braucht, um die Umklassifizierung zu bewirken. Ich habe bei gleichgrossen Personen, die z. B. bei $171 \mathrm{~cm}$ Scheitclsohlenlänge im nzentralnormalen" Ernährungszustand $(73 \mathrm{~kg}$ ) ganz das gleiche Brustumfangsmittel von $96 \mathrm{~cm}$ hatten, im nsehr mageren "Zustand (49 kg) $87 \mathrm{~cm}$, im nsehr fetten" Zustand $(129,7 \mathrm{~kg}) 123 \mathrm{~cm}$ Brustumfangsmittel - also Unterschicde von $36 \mathrm{~cm}$ (!) gefunden. Was hier rom absoluten Brustumfangsmass gesagt ist, gilt auch für das "proportionelle".

Ich komme damit zu dem Schluss: Weder die Körpergewichte, noch die Körperlängen, noch die Brustumfänge sind bei der von Brugsch angewandten Messungsart geeignet, $n$ verschleierte Notwendigkeiten" in den Beziehungen dieser Maasse zu einander zu enthüllen. Ein Blick auf meine Kurve 1, weiche zum Vergleich die Brugsch'sche Kurve 5 mit enthält, kann das Gesagte nur bestätigen. Auch bei anthropologischen Habitusuntersuehungen, die Körpergewichte und Brustumfänge betreffen, kann die Berücksichtigung des abgestuften Ernährungszustandes nicht unterbleiben, ohne zu fehlerhaften Schlussfolgerungen zu führen. Für die Findung formelmässiger Beziehungen zwischen Körperlänge und Körpergewicht ist ausserdem die „Oberlänge ${ }^{u}$ zu berücksichtigen. Der kleine Spielraum, den ich zwischen den zentralnormalen Formel- und Istgewichten durchschnittlich auf $\pm 3,5 \mathrm{~kg}$ bemessen habe, weil der höchste Gewichtsunterschied bei gleichgrossen Einzelpersonen für meine Männer etwa $7 \mathrm{~kg}(= \pm 3,5)$ betrug, stellt in 95 pCt. aller Fälle die Variationsbreite der "zentralnormalen ${ }^{\prime}$ Istgewichte gleichgrosser Einzelpersonen dar, die erzeugt ist durch Knochen-Muskel-Eingeweidefüllungs-Schulterbreiten-Differenzen. Brugsch scheint nach alledem teilweise von Voraussetzungen ausgegangen zu sein, deren Richtigkeit er nicht "ceteris paribus" geprüft haben kann. Nur nebenbei möchte ich noch bemerken, dass ich es auch nicht für glücklich balte, wenn Brugsch seine Kurve 1, die eine unverkennbare "Variationskurve der Häufigkeit im Vorkommen der einzelnen Körperlängenstufen " ist, "kurz" mit "Variationen der Körperlänge" bezeichnet und diese Kurve bei Kurve 2 "auflöst" in drei "Körperlängengruppen mit Körperlängengipfeln ${ }^{u}$ statt in ${ }_{n}$ Häufigkeitsgruppen mit Häufigkeitsgipfeln".

\section{Entgegnung zu der voranstehenden Arbeit von Dr. Oeder.} Von

\section{Prof. Theodor Brugseh.}

Eine ins einzelne gehende Erwiderung auf Oeder's Ausführungen kann ich mir ersparen, da Oeder's Feststellungen nicht das treffen, was man „Normalgewicht" ${ }^{4}$ nennen muss. Normal ${ }^{1}$ ) heisst nichts weiter als

1) cf. z. B. Muther, Wörterbuch der Philosophie. 
Durchschnitt oder Mitte. Man kann den Durchschnitt eng oder weit nehmen, kann - wenn man ein ganzes Land durchuntersuchen könnte den Durchschnitt aus allen Körpergewichten der Eiswohner nehmen, oder wenn man sieh auf eine Populationsgruppe beschrïnt, den Durchschnitt aus dieser ziehen, man kann den Durchschnitt noch weiter verengern, indem man cine bestimmte Altersklasse oder eine bestimmte soziale Klasse usw. wählt. Ich wählte eine Altersklasse socben ausgewachsener (nicht unerwachsener!) Individuen gleicher Population und bestimmte zu jeder Längengruppe das Durchschnittsgewicht. Dass dieses sich nicht auf eine allgemein gültige Formel bringen lässt, ist eine Tatsache, an der alle Ausfübrungen Oeder's nichts ändern können.

Oeder erklärt ein Individuum für normal im Ernührungszustand, das weder mager noch fett erscheint, auf Grund von Kriterien, die ich für unsere medizinische Beurteilung im gewissen Sinne anerkenne ${ }^{1}$ ). Will er aber Körpergewichte solcher Individuen als Normalgewichte aufstellen, so stellt er sich ausserhalb des Rahmens des Sprachgebrauchs und der Wissenschaft. Nun geht aber Oeder noch weiter: er stellt eine Formel zur Berechnung eines solches Normalgewichtes auf und sucht zu beweisen, dass Formelgewicht und Istgewicht übereinstimmen. Ich frage wozu? Wenn ich nach Oeder's Kriterien durch Aspekt und durch Dickenmessung einer Hautfalte am Nabel feststellen kann, ob ein Individuum im Oeder'schen Sinne einen "normalen" Ernährungszustand aufweist, dann brauche ich wirklich keine Formel. Das Klagelied, das Oeder über die Abneigung, die man gegen seine Formel hat, anstimmt, ist das Wiegentied über ein totes Kind! Ich halte es aber geradezu für gefährlich, eine Formel im Ooder'sehen Sinne zu akzeptieren. Oeder's Material scheint sich aus sozial gut gestellten, daher gut genährten Individuen mittlerer Jahro zu rekrutieren, so wie sie eben eine Diätkuranstalt aufsuchen kömnen, wobei die Patienten (vor dem Kriege!) wohl mehr minder überernährt waren. Ein Jugendlicher ist aber nach Oeder's Formulierung mager; je älter das Individuum, desto mehr setat es Fett an (siehe die Hassing'sche Tabelle!). Das lässt sich alles nicht durch eine Formel ausdrücken und würde nur eine Vergewaltigung darstellen. Normalgewicht ist Durchschnittsgewicht und dabei muss es bleiben. Ueber meine anthropologischen Untersuchungen mich mit Oeder auseinanderzusetzen, erscheint mir nicht notwendig.

1) Der Orientale würde schon andere Gesichtspunkte der Beurteilung haben. 